\title{
RhoA is associated with invasion and poor prognosis in colorectal cancer
}

\author{
DONGJUN JEONG $^{1 *}$, SOYOUNG PARK $^{1 *}$, HYUNGJOO KIM ${ }^{1}$, CHANG-JIN KIM ${ }^{2}$, TAE SUNG AHN ${ }^{3}$, \\ SANG BYUNG BAE ${ }^{4}$, HAN JO KIM ${ }^{4}$, TAE HYUN KIM ${ }^{5}$, JUNGKYUN IM ${ }^{6}$, \\ MOON SOO LEE ${ }^{3}$, HYOG YOUNG KWON ${ }^{7}$ and MOO JUN BAEK ${ }^{3}$ \\ ${ }^{1}$ Soonchunhyang Medical Science Research Institute, Departments of ${ }^{2}$ Pathology, ${ }^{3}$ Surgery and ${ }^{4}$ Oncology, \\ College of Medicine, Soonchunhyang University; Departments of ${ }^{5}$ Chemistry and ${ }^{6}$ Nanochemical Engineering, \\ ${ }^{7}$ Soonchunhyang Institute of Medi-bio Science, Soonchunhyang University, Cheonan, Chungcheongnam-do, Republic of Korea
}

Received October 13, 2015; Accepted November 13, 2015

DOI: $10.3892 /$ ijo.2015.3281

\begin{abstract}
Colorectal cancer is one of the most common cancers and is the fourth leading cause of cancer death in Korea. Mortality of colorectal cancer is strongly associated with the metastatic spread of the disease. As such, it is important to find and characterize signaling pathways involved in colon cancer metastasis. We investigated the functional importance of RhoA using human cell lines as well as 150 colorectal cancer patient-derived samples as it remains unclear whether RhoA functions as either an oncogene or a tumor suppressor in colon cancer. RhoA was highly expressed in metastatic cancer cell lines. Although cancer cell proliferation was only moderately impaired after depletion of RhoA, RhoA-depleted cancer cells exhibited markedly reduced migration and invasion ability in vitro. Furthermore, we found that RhoA is associated with the invasion of lymph nodes and blood vessels in the patient colorectal cancer samples. Most notably, patients with higher RhoA expression had a significantly poorer 5-year survival rate after surgery. These results suggest that RhoA is a marker of poor prognosis in colorectal cancer and may be a promising target for cancer treatment.
\end{abstract}

\section{Introduction}

Colorectal cancer is one of the most common type of cancers and is the fourth leading cause of cancer deaths in Korea (1). In

Correspondence to: Dr Hyog Young Kwon, Soonchunhyang Institute of Medi-bio Science, Soonchunhyang University, 25 Bongjeong-ro, Dongnam-gu, Cheonan-si, Chungcheongnam-do, Republic of Korea E-mail: hykwon@sch.ac.kr

Dr Moo Jun Baek, Department of Surgery, College of Medicine, Soonchunhyang University, 31 Soonchunhyang 6 gil, Dongnam-gu, Cheonan, Chungcheongnam-do, Republic of Korea

E-mail: ssurge@schmc.ac.kr

${ }^{*}$ Contributed equally

Key words: RhoA, colorectal cancer, prognosis, metastasis addition, the incidence rates of colorectal cancer have continued to increase in both genders from 1999 to 2010 (1). Despite advances in surgical resection and systemic chemotherapies, the most important factors contributing to progression and poor prognosis of cancer are recurrence and metastasis (2-4). However, the signaling pathways involved in tumor development, progression, and metastasis are highly complex and require further characterization. Therefore, it is essential to identify and investigate signaling pathways involved in cancer recurrence or metastasis of colorectal cancer.

Rho GTPases belong to the small GTPase family of proteins $(\sim 21 \mathrm{kDa})$ that includes Ras, Rab, Arf, and Rho families $(5,6)$. These proteins have been implicated in many important cancer-related processes in mammalian cells, such as proliferation, migration, and survival. Mammalian cells express more than 22 Rho GTPases, such as Rho isoforms (A, B and C), three Rac isoforms (1, 2 and 3), Cdc42, among others $(7,8)$. Rho GTPases exist in two forms: the inactive, GDP-bound form, or the active, GTP-bound form. This dynamic form allows these proteins to function as molecular switches when activated by cell surface receptors, leading to transcriptional activation, cytoskeleton reorganization, and cell migration (9). Rho GTPases are highly expressed or activated in multiple cancers (7); RhoA inhibition using small interfering RNA has been shown to reduce proliferation and tumor burden in vitro and in vivo (10-12). Additionally, RhoA expression is higher in tumor samples than in normal tissues (13). However, recently, it has been suggested that RhoA acts as a tumor suppressor in colorectal cancer, suppressing tumor progression and metastasis (14). Thus, it is still unclear whether RhoA functions as a promoter or suppressor of colorectal cancer.

\section{Materials and methods}

Ethics statement. All procedures performed in studies involving human participants were in accordance with the ethical standards of Ethics Committee of Soonchunhyang University Cheonan Hospital and with the 1964 Helsinki declaration and its later amendments or comparable ethical standards. Informed consent was obtained from all individual participants included in the study. 
Cell lines. Human colorectal cancer cell lines HCT116, HT29, LoVo, SW480, SW620, colo201, colo205, and $\mathrm{CaCO}_{2}$ were purchased from the Korean Cell Line Bank (KCLB). Cells were grown in RPMI-1640 medium (Cellgro, USA) supplemented with $10 \%$ fetal bovine serum (Equitech-bio, USA) and $1 \mathrm{X}$ penicillin/streptomycin (Cellgro) at $37^{\circ} \mathrm{C}$ in a humidified atmosphere containing $5 \% \mathrm{CO}_{2}$.

Plasmid constructs and transfection. Short hairpin RNA (shRNA) constructs were designed and cloned into the H1-shRNA vector (Genolution Pharmaceuticals Inc.). The target sequence for RhoA is 5'-CAGAAAAGTGGACCC CAGAA-3'. The sequence of nonsense shRNA against luciferase was provided by Genolution Pharmaceuticals Inc. The plasmids were transfected into HCT116 cells using Lipofectamine 2000 (Invitrogen, USA) according to the manufacturer's instructions. Briefly, HCT116 cells were cultured and transfected using Lipofectamine 2000. Medium was changed $6 \mathrm{~h}$ after transfection, and transfected cells were selected using Zeocin (Invitrogen). To evaluate transfection efficiency, semi-quantitative reverse transcriptase-polymerase chain reaction (RT-PCR) was used.

$R N A$ extraction and RT-PCR. RNA was isolated using TRIzol (Invitrogen); equal amounts of RNA were converted to cDNA using ReverTra Ace ${ }^{\circledR}$ qPCR kit (Toyobo, Japan) according to the manufacturer's instructions. To determine RhoA expression, PCR was performed using the Maxime PCR PreMix kit (iNtRON, Korea). Primer sequences are as follows: RhoA-F, 5'-CATCCGGAAGAAACTGGT-3'; RhoA-R, 5'-TCCCAC AAAGCCAACTC-3'; GAPDH (glyceraldehyde-3-phosphate dehydrogenase)-F, 5'-CTTAGCACCCCTGGCCAAG-3'; GAPDH-R, 5'-GATGTTCTGGAGAGCCCCG-3'; Zeocin-F, 5'-CGACGTGACCCTGTTCATCAG-3'; Zeocin-R, 5'-GTT CGTGGACACGACCTCCGA-3'. The expected amplicon sizes were $168 \mathrm{bp}$ (RhoA), 121 bp (GAPDH), and $130 \mathrm{bp}$ (Zeocin). The PCR cycles consisted of a pre-denaturation step at $95^{\circ} \mathrm{C}$ for $10 \mathrm{~min}$, followed by 353 -temperature cycles $\left(95,59.5\right.$ and $\left.72^{\circ} \mathrm{C}\right)$ for $30 \mathrm{sec}$ each, and a final extension at $72^{\circ} \mathrm{C}$ for $5 \mathrm{~min}$. The PCR products were confirmed using the QIAxcel auto electrophoresis system (Qiagen, USA).

Western blot analysis. Cell lysates were harvested using PRO-PREP (iNtRON, Korea) for $30 \mathrm{~min}$ on ice and centrifuged at $13,000 \mathrm{rpm}$ for $5 \mathrm{~min}$ at $4^{\circ} \mathrm{C}$. Protein concentration of the supernatant was determined by BCA assay (Thermo, USA). An equal amount of each protein extract $(50 \mu \mathrm{g})$ was resolved using $10 \%$ polyacrylamide gel and electrotransferred onto $0.2 \mu \mathrm{m}$ polyvinylidene fluoride (PVDF) membrane (Millipore, USA) using Trans-blot turbo (Bio-Rad Laboratories, Inc., USA). Membranes were immunoblotted with either 1:1,000-diluted mouse anti-RhoA monoclonal antibody (Abnova, Taiwan) or 1:5,000-diluted mouse anti$\beta$-actin monoclonal antibody (Sigma, USA) overnight at $4^{\circ} \mathrm{C}$. Membranes were incubated with 1:10,000-diluted horseradish peroxidase-conjugated anti-mouse immunoglobulin (Sigma) for $1 \mathrm{~h}$ at room temperature. The protein signal was detected by enhanced chemiluminescence (Advansta, USA) using the Molecular Imager ChemiDoc XRS+ System (Bio-Rad Laboratories, Inc.).
Cell proliferation assay (MTT assay). MTT assay was used to evaluate cell proliferation after transfection. Cells $\left(1.0 \times 10^{5}\right.$ cells/well) were seeded into a 96-well plate and incubated for an additional 24-72 h post-transfection. After time-dependent incubation, the medium was removed and the cells were washed with PBS. The cells were incubated in a $5-\mathrm{mg} / \mathrm{ml}$ MTT (Sigma) solution for $4 \mathrm{~h}$. Then, the media was substituted with dimethyl sulfoxide (DMSO; Sigma) and placed on the plate shaker for $15 \mathrm{~min}$. Absorbance was read at $570 \mathrm{~nm}$ using a plate reader.

Migration assay (wound healing assay). Cell migration was analyzed in vitro using the Culture insert system 24 (ibidi, Germany). The culture insert was attached to the bottom of a 6-well plate, and $100 \mu \mathrm{l}$ of media containing $1.0 \times 10^{6}$ cells were seeded into each well of the insert. The culture inserts were removed from the plate after $48 \mathrm{~h}$, and cells were further cultured with fresh RPMI-1640 medium contained 10\% FBS. The cell gap was monitored every $12 \mathrm{~h}$ for $48 \mathrm{~h}$. Cell images were taken every $12 \mathrm{~h}$ with a phase contrast microscope, AxionCam camera (Zeiss, Germany).

Invasion assay (Matrigel invasion assay). The transwell culture insert was pre-coated with $50 \mu 1$ of Matrigel (BD, USA) according to the manufacturer's instructions. Cells $\left(5.0 \times 10^{6}\right)$ were suspended in serum-free RPMI-1640 medium and seeded into the pre-coated insert. Eight hundred microliters of RPMI-1640 medium containing 10\% FBS was added outside the transwell culture insert. Cells were incubated at $37^{\circ} \mathrm{C}$ for $24 \mathrm{~h}$ in a humidified atmosphere with $5 \% \mathrm{CO}_{2}$. After washing the transwell insert twice with PBS, cells were fixed with $10 \%$ formaldehyde for $2 \mathrm{~min}$. The cells were permeabilized with methanol for $20 \mathrm{~min}$ and then stained with methylgreen for $15 \mathrm{~min}$. The transwell insert was washed twice with PBS, wiped using cotton swab, and then observed using an inverted microscope.

Immunofluorescent staining. Immunostaining was conducted as previously described, with slight modification, to determine morphology change after transfection (15). Briefly, $1 \times 10^{5}$ cells were seeded on a cover slip placed in a 6-well plate and incubated at $37^{\circ} \mathrm{C}$ in a humidified atmosphere with $5 \% \mathrm{CO}_{2}$ for $24 \mathrm{~h}$. The wells were washed twice with cold PBS and fixed in cold methanol/acetone $1: 1(\mathrm{vol} / \mathrm{vol})$ for $30 \mathrm{~min}$ at $-20^{\circ} \mathrm{C}$. The methanol/acetone mixture was then removed and the wells were dried for $15 \mathrm{~min}$ at room temperature. After rehydration with PBS for $15 \mathrm{~min}$ at room temperature, cells were incubated with blocking buffer $(2 \mathrm{mg} / \mathrm{ml}$ BSA in PBS) for $1 \mathrm{~h}$ at room temperature, and then rinsed with blocking buffer three times. Cells were stained with $1 \mu \mathrm{g} / \mathrm{ml}$ DAPI for $2 \mathrm{~min}$, sealed with clear nail polish, and visualized using an Olympus FV10 confocal microscope.

Human colorectal carcinoma specimens. A total of 150 colorectal carcinoma tissue specimens were obtained from Soonchunhyang University Cheonan Hospital, Korea, where samples were collected from patients who underwent surgery between 2002 and 2007. These tissues were formalin-fixed and paraffin-embedded (FFPE). Clinicopathological data including age, gender, TNM classification, and distal metastasis 
are shown at Table I. Tumor stage was identified according to the American Joint Committee on Cancer TNM classification system. Sample collection for this study was approved by the Ethics Committee of Soonchunhyang University, Cheonan Hospital.

Tissue microarray (TMA) and immunohistochemistry. Immunohistochemical staining was performed using tissue microarray (TMA) block sections to determine RhoA expression in patient samples. The FFPE tumor tissues were re-embedded from each FFPE block to the recipient block in duplicate. Each TMA block contained 60 cores from 30 samples. For immunohistochemistry, 4- $\mu \mathrm{m}$ sections were obtained using a microtome, deparaffinized in xylene, and rehydrated in 100-70\% alcohol series. Antigen retrieval was achieved in citrate buffer $(\mathrm{pH}$ 6.0) using a microwave for $15 \mathrm{~min}$. To eliminate endogenous peroxidase activity, the sections were incubated in peroxidase blocking solution (Dako, Denmark) for $30 \mathrm{~min}$ and then washed with phosphate-buffered saline containing 0.1\% Tween-20 (PBST). The sections were incubated with anti-mouse RhoA antibody (Abnova, 1:500) for $2 \mathrm{~h}$ at room temperature, followed by incubation in enhancer for $30 \mathrm{~min}$ and treatment with polymer for $1 \mathrm{~h}$ at room temperature. After washing with PBST, sections were incubated with DAB, counterstained with hematoxylin, and observed under a microscope.

IHC data analysis. The RhoA-stained tissue cores were examined by 2 independent observers (Chang-Jin Kim and Dongjun Jeong), and a consensus score was determined for each specimen. A positive reaction was scored into 4 grades, according to the intensity of the staining: $0,1+, 2+$, and $3+$. The percentages of RhoA-positive cells were also scored into 4 categories: 0 (0\%), 1 (1-30\%), 2 (31-70\%) and 3 (71-100\%). The final score, calculated as the product of the intensity score multiplied by the percentage score, was classified as follows: 0 for negative; 1-3 for weak; 4-6 for moderate; and 7-9 for strong. Samples with a final score $\leq 3$ were grouped together as RhoA expression negative while those with a score $\geq 4$ were grouped together as RhoA expression positive.

Statistical analysis. Statistical analysis was conducted using SPSS 19.0 (Chicago, IL, USA) program. The results of RT-PCR, western blotting, and functional characterization of cells were analyzed with one-way ANOVA test and Student's t-test. The relationship between the result of immunohistochemistry and clinicopathological data was analyzed with Chi-square test. Hazard ratio and $95 \%$ confidence interval were evaluated using Cox regression models. Kaplan-Meier method was used to analyze disease-free survival rate using the log-rank test. A $\mathrm{p}$-value of $<0.05$ was considered statistically significant in all assessments.

\section{Results}

RhoA expression in various colorectal carcinoma cell lines. As RhoA has been implicated in multiple cancers including breast cancer, liver cancer, ovarian carcinoma, and gastric carcinoma, we examined the expression of RhoA in both non-metastatic and metastatic colorectal cancer cell lines. A
Table I. Clinicopathological features of patient samples.

\begin{tabular}{|c|c|}
\hline Clinicopathological factors & $\mathrm{N}$ \\
\hline \multicolumn{2}{|l|}{ Sex } \\
\hline Male & 93 \\
\hline Female & 57 \\
\hline \multicolumn{2}{|l|}{ pT stage } \\
\hline 1 & 4 \\
\hline 2 & 22 \\
\hline 3 & 108 \\
\hline 4 & 16 \\
\hline \multicolumn{2}{|l|}{ pN stage } \\
\hline 0 & 76 \\
\hline 1 & 48 \\
\hline 2 & 26 \\
\hline \multicolumn{2}{|l|}{ Distal metastasis } \\
\hline Negative & 144 \\
\hline Positive & 6 \\
\hline \multicolumn{2}{|l|}{ Vascular invasion } \\
\hline Negative & 123 \\
\hline Positive & 27 \\
\hline \multicolumn{2}{|l|}{ Lymphatic invasion } \\
\hline Negative & 118 \\
\hline Positive & 32 \\
\hline \multicolumn{2}{|l|}{ Clinical stage } \\
\hline I & 18 \\
\hline II & 56 \\
\hline III & 70 \\
\hline IV & 6 \\
\hline
\end{tabular}

Total of 150 cases of colorectal cancer patient samples were used in this study. Tumor stage was classified according to the American Joint Committee on Cancer tumor-node-metastasis (TNM) classification.

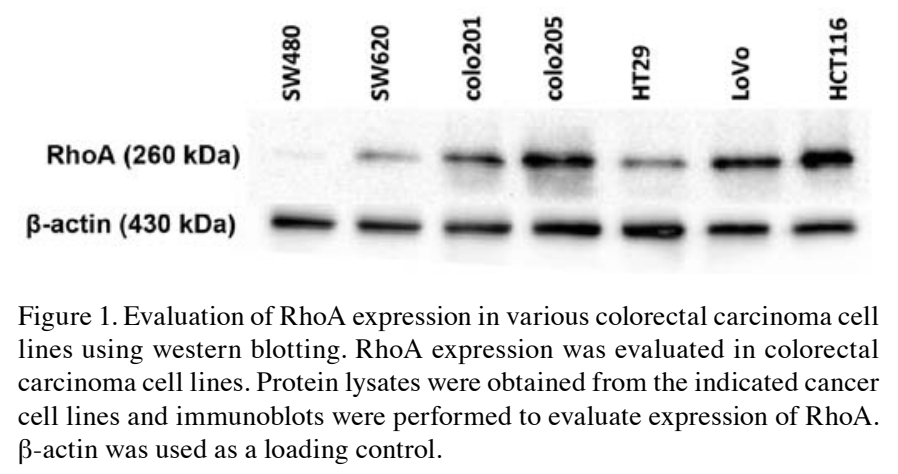

non-metastatic cancer cell line, SW480, expressed a very small amount of RhoA, whereas many cancer cell lines including colo205, LoVo, and HCT116 exhibited high levels of RhoA expression (Fig. 1). Notably, the metastatic cell line HCT116, 
A

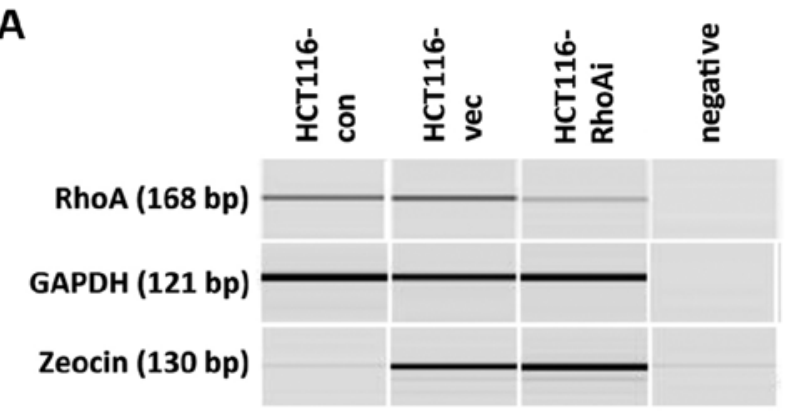

B

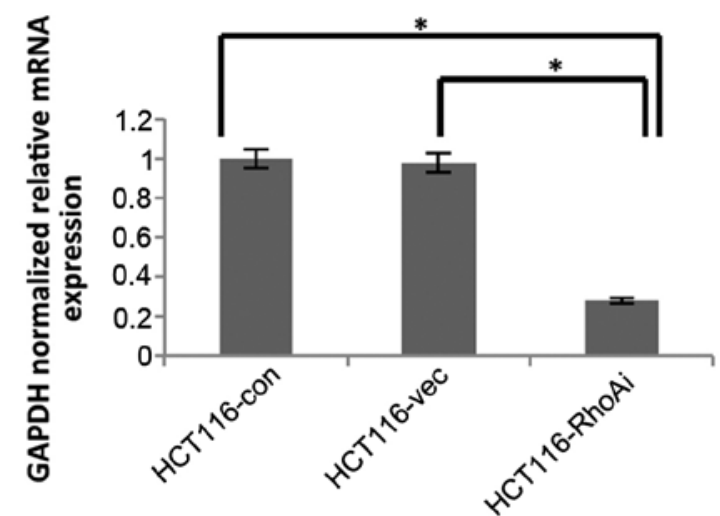

C

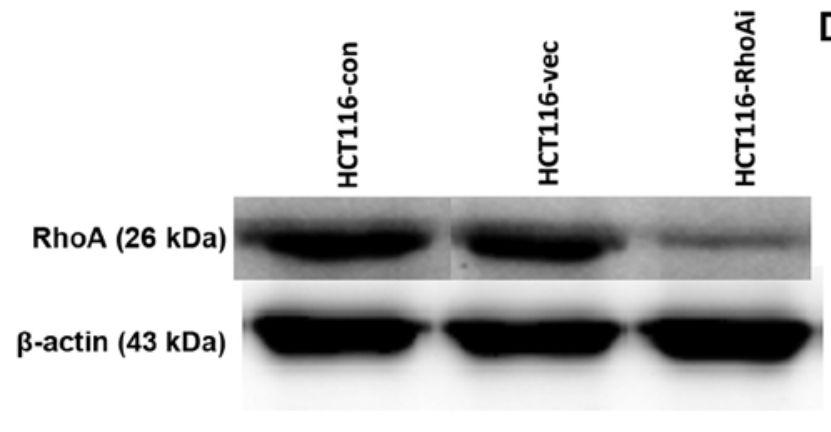

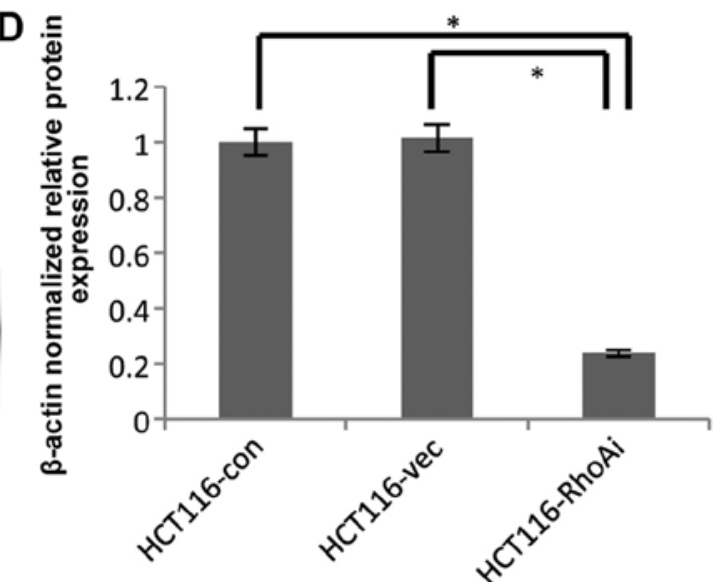

Figure 2. Knockdown efficiency of RhoA in HCT116 cells. HCT116 cells were untransfected (HCT116-control) or transfected with either control vector (HCT116-vector) or shRhoA (HCT116-RhoAi). RNA and protein were isolated to assess expression of RhoA. (A and B) RT-PCR was performed to determine RhoA mRNA expression in HCT116 cells after transfection. (A) A representative image of RT-PCR results is shown. (B) RhoA expression is normalized to GAPDH mRNA. Data shown are from three independent experiments (HCT116-control and HCT116-RhoAi group, $\mathrm{p}=0.028$; HCT116-vec and HCT116RhoAi group, $\mathrm{p}=0.035$; ${ }^{*} \mathrm{p}<0.05$ ). ( $\mathrm{C}$ and $\mathrm{D}$ ) Immunoblotting with anti-RhoA Ab was conducted to assess RhoA protein level. (C) A representative result of immunoblotting is shown. (D) The protein level of RhoA is normalized to $\beta$-actin. Data shown are from three independent experiments (HCT116-control and HCT116-RhoAi group, $\mathrm{p}<0.0001$; HCT116-vec and HCT116-RhoAi group, $\mathrm{p}<0.0001$; ${ }^{\mathrm{p}} \mathrm{p}<0.0001$ ).

had the highest RhoA protein expression among the cell lines tested. Therefore, we used the HCT116 cancer cell line for further studies to determine the function of RhoA in the carcinogenesis of colon cancer.

shRNA-mediated RhoA knockdown in the HCT116 cell line. To determine the functional consequence of RhoA, we used shRNA to knock down RhoA in HCT116 cells. The knockdown efficiency of RhoA was confirmed using RT-PCR and immunoblotting (Fig. 2). Specifically, HCT116 cells were transfected with either nonsense control (HCT116-vec) or shRhoA (HCT116-RhoAi). RNA and protein lysates from each pair of transfected cells were extracted and determined to assess RhoA levels. To determine the knockdown efficiency at the RNA level, RT-PCR was performed using cDNA from the samples. Amplicon size is $168 \mathrm{bp}$ for RhoA, $121 \mathrm{bp}$ for GAPDH, and $130 \mathrm{bp}$ for zeocin. The PCR products were analyzed with the auto electrophoresis system, QIAxcel (Qiagen, USA). Zeocin was used to compare the transfection efficiency between nonsense control and shRhoA, and indicated similar transfection efficiencies (Fig. 2A). The expression of RhoA was significantly downregulated in shRhoA-treated HCT116 cells (HCT116-RhoAi) compared to untransfected (HCT116-con) and nonsense control (HCT116-vec) cells $(\mathrm{p}=0.028$ and $\mathrm{p}=0.035$, respectively). Specifically, the RhoA RNA level decreased by $\sim 70 \%$ after shRhoA transfection, and there was no difference between the untransfected group (HCT116-con) and the control transfected group (HCT116-vec) (Fig. 2B).

As changes at RNA level do not always correlate with changes at the protein level, it is necessary to determine the protein level after knockdown. To test this, we also determined RhoA protein level after shRNA-mediated depletion. Protein lysates from each pair of transfected cells were collected and immunobloted with anti-RhoA Ab (Fig. 2C and D). Protein expression also displayed the same pattern observed in RNA after knockdown of RhoA. The protein level of RhoA was decreased by $70 \%$ in HCT116-RhoAi compared to untransfected cells or vector-transfected cells $(\mathrm{p}<0.0001)$. These results led us to confirm that RhoA was successfully knocked down.

Knockdown of RhoA impairs the proliferation of HCT116 cells. To functionally define the role of RhoA, we first determined proliferation ability in the HCT116 cell line after RhoA knockdown using an MTT assay. After transfection of either control vector or shRhoA, cells were incubated for different time periods, ranging from 24 to $72 \mathrm{~h}$, to determine proliferation rate. As shown in Fig. 3A, the proliferation of shRhoA-trans- 

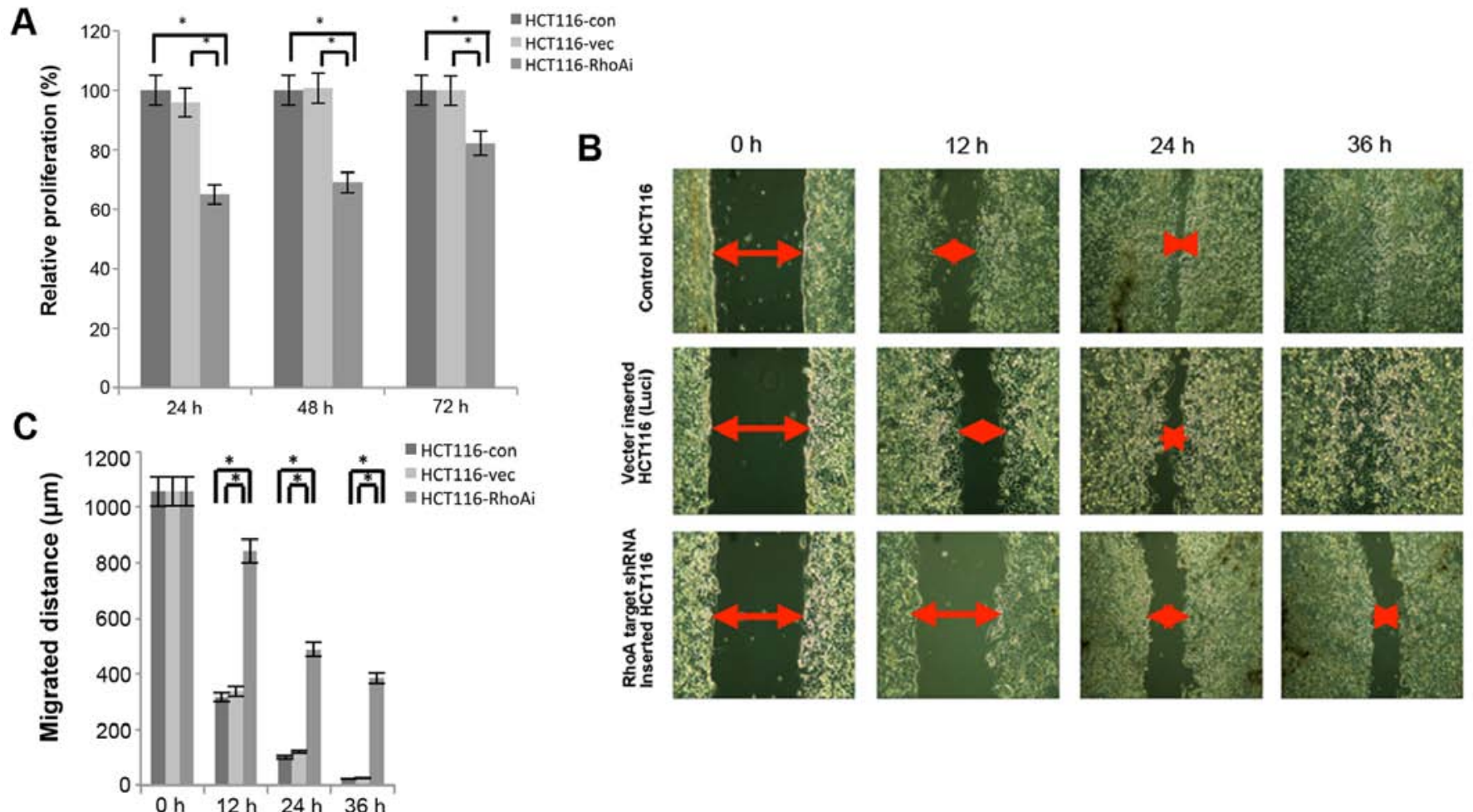

Figure 3. RhoA depletion impairs cell proliferation and migration in the HCT116 cell line. (A) HCT116 cells were transfected with either control vector or shRhoA and incubated between 24 and $72 \mathrm{~h}$. Cell proliferation was determined by MTT assay. Statistical significance was analyzed using one-way ANOVA ( ${ }^{*}<<0.05$; HCTT116-control group and HCT116-RhoAi group, $\mathrm{p}<0.0001$; HCT-vec and HCT116-RhoAi, $\mathrm{p}=0.002$ ). (B and C) HCT116 cells untransfected or transfected with either control vector or shRhoA were wounded at hour 0 and then cultured in RPMI-1640 medium supplemented with $10 \%$ fetal bovine serum. Gap distance was monitored and cell migration images were taken every $12 \mathrm{~h}$ with an inverted microscope (magnification, $\mathrm{x} 400$ ). (B) A representative image of cell migration is shown. (C) Data shown are from three independent experiments. Statistical significance was assessed using one-way ANOVA ( $\mathrm{p}<0.00001)$.

fected cells was lower than that of HCT116-con or HCT116-vec cells $(24 \mathrm{~h}, \mathrm{p}<0.0001 ; 48 \mathrm{~h}, \mathrm{p}<0.0001 ; 72 \mathrm{~h}, \mathrm{p}=0.002)$. The proliferation between HCT-con and HCT116-vec cells was not significantly different. These results suggest that RhoA plays a role in proliferation of colon cancer cells.

RhoA is essential for migration of HCT116 cells. In order to assess the effect of RhoA on migration of HCT116 cells, migration ability was determined after knocking down RhoA using a wound healing assay. Cells were seeded into culture insert, which was attached to the bottom of a 6-well plate, and the culture inserts were removed two days later. The cells were further cultured and monitored every $12 \mathrm{~h}$ (Fig. 3B and C). Over time, cells in control groups migrated gradually. However, the shRhoA-transfected group displayed approximately a 2-fold delay in migration at $12 \mathrm{~h}$ and a 3 -fold delay at $24 \mathrm{~h}$ compared to control groups. More significantly, while control groups showed almost no gaps at $36 \mathrm{~h}$, shRhoA group still displayed $\sim 400-\mu \mathrm{m}$ gaps, suggesting that RhoA-deficient cells were significantly impaired in the ability to migrate (Fig. 3C).

RhoA is required for invasion in HCT116 cells. As previous results showed that Rho is essential for migration in HCT116 cells, we aimed to determine whether RhoA is also required for invasion. To achieve this goal, HCT116 cells were transfected and seeded on a Matrigel-coated insert. Cells were then incubated for an additional $48 \mathrm{~h}$ after seeding, and cells were imaged to assess cell invasion. HCT116 cells transfected with shRhoA exhibited significantly less invasion than control cells (Fig. 4A). Thus, we examined the morphological characteristics of HCT116 cells following RhoA knockdown. To assess cell morphology, nuclei of RhoA-depleted cells were stained with DAPI and analyzed with a confocal microscope. Vector-transfected or untransfected cells exhibited elongated morphology with pseudopodia, whereas RhoA-depleted cells were more rounded with less pseudopodia, and multinucleated in $\sim 20 \%$ cells tested (Fig. 4B). These results indicate that RhoA is essential for cell migration and invasion of colon cancer cells, two important factors contributing to mortality caused by colon cancer.

RhoA expression is associated with a poor prognosis in colorectal cancer. Based on the conclusion that RhoA is essential for the migration and invasion in the HCT116 cell line, we then analyzed RhoA expression in patient colorectal cancer samples to define whether RhoA is relevant in human disease. The samples, obtained from 150 patients who had undergone surgery, were stained with RhoA antibody to determine RhoA expression. RhoA protein stained primarily in the cytoplasm with a wide range of intensity; RhoA stain intensity was graded from negative to strong expression (Fig. 5A-C and Table II). RhoA expression was confirmed to be positive in 86 cases out of 150 samples (57.3\%). We further correlated RhoA expression with several factors including age, gender, $\mathrm{pN}$ stage, metastasis, and invasion, among others. RhoA expression was not significantly correlated with age, gender, and $\mathrm{pN}$ stage 
A

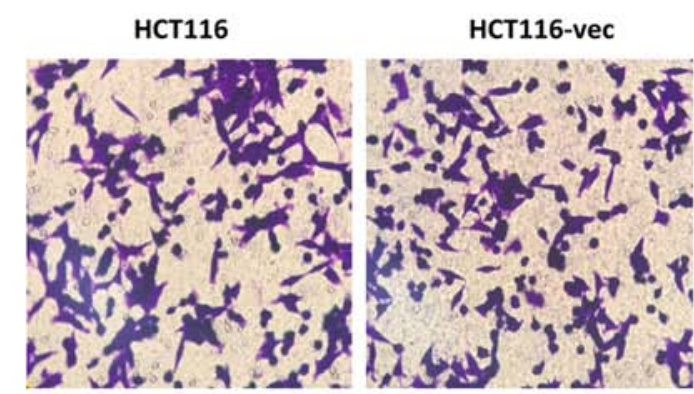

HCT116-con

B

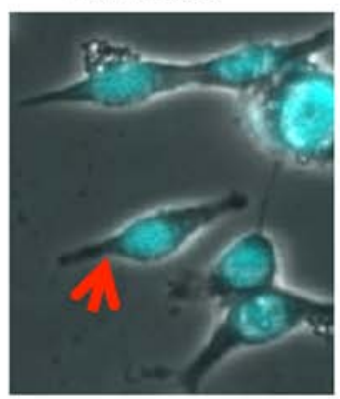

HCT116-vec

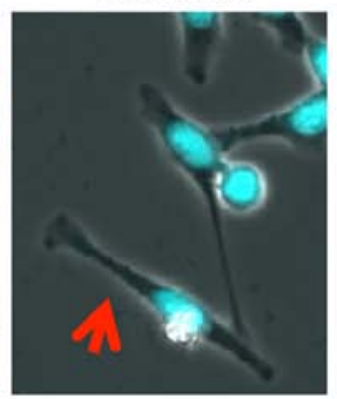

HCT116-shRNA-RhoAi

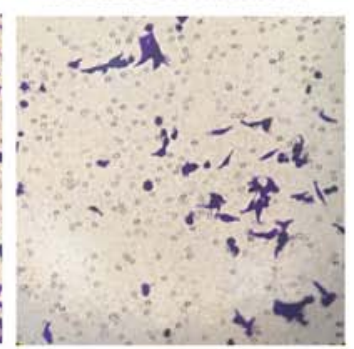

HCT116-RhoAi

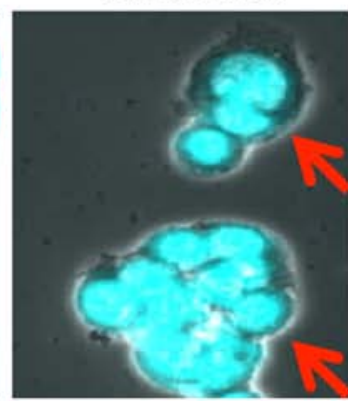

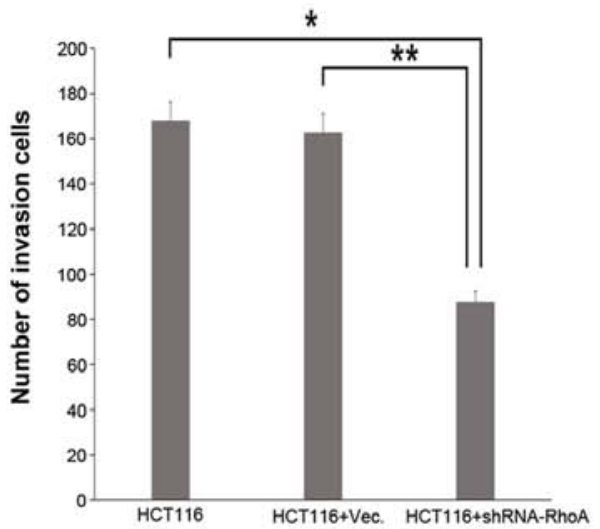

Figure 4. RhoA is required for invasion in the HCT116 cell line. (A) HCT116 cells untransfected (HCT116-con) or transfected with either control vector or shRhoA were seeded in a Matrigel-coated transwell. The invasive activity of cells was quantified $48 \mathrm{~h}$ after seeding. Cell invasion images were taken with an inverted microscope (magnification, $\mathrm{x} 400)$. Statistical significance was assessed using one-way ANOVA ( $\left.{ }^{*} \mathrm{p}<0.05 ;{ }^{* *} \mathrm{p}=0.001\right) .(\mathrm{B}) \mathrm{Morphology}$ changes in HCT116 cells after RhoA knockdown. The nucleus of HCT116 cells was stained with DAPI after transfection to assess potential changes in cell morphology. Cell images were taken with an Olympus FV10 confocal microscope (magnification, x600).

A

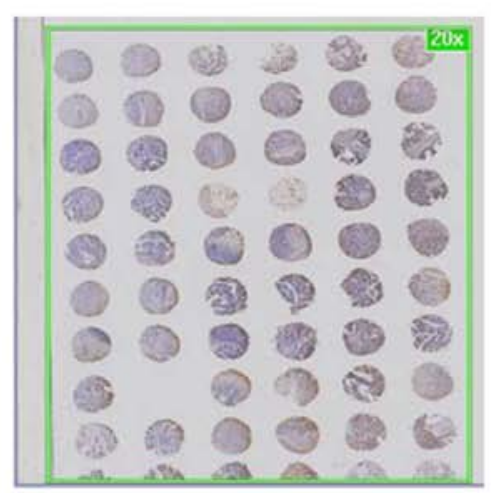

B

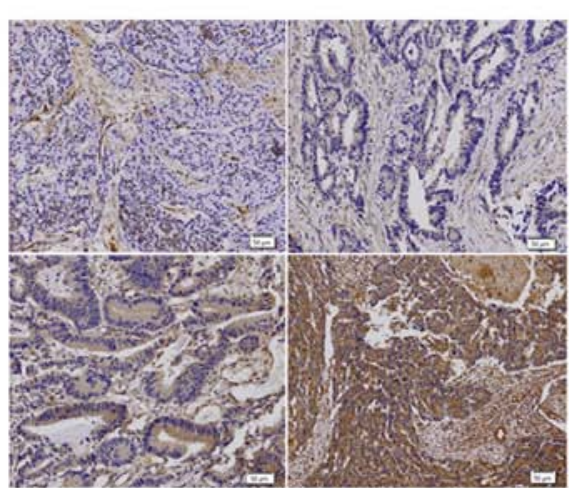

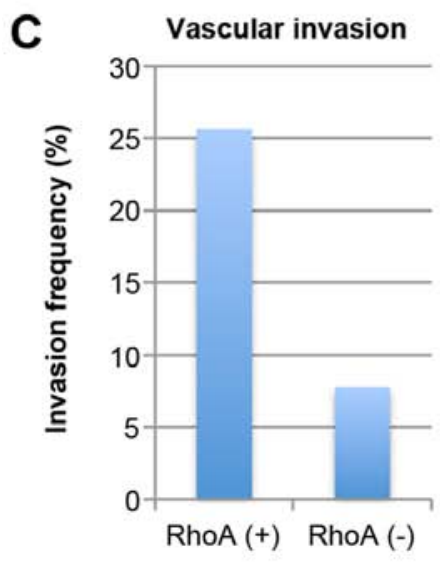

D

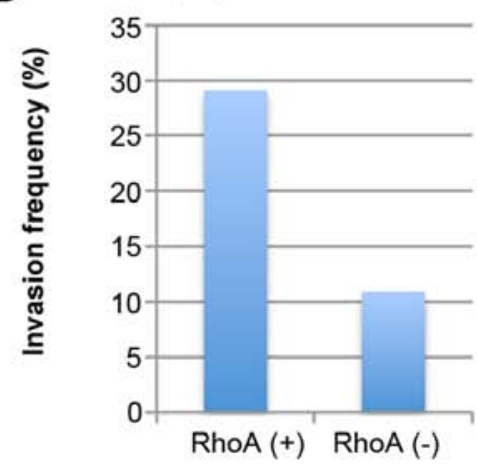

E

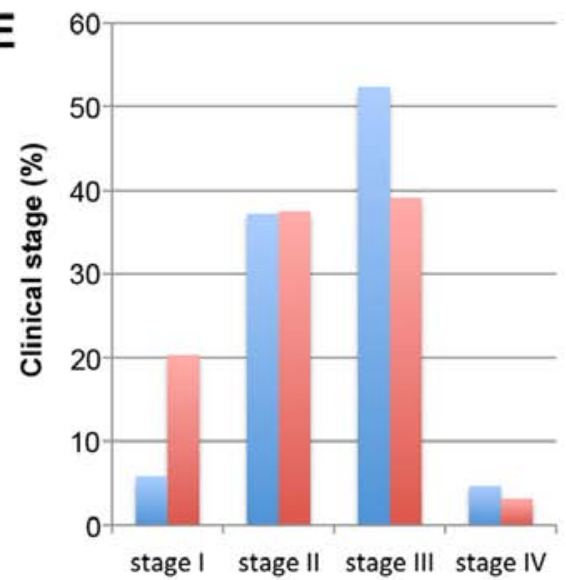

$=$ RhoA(+)

$=\mathrm{RhoA}(-)$

Figure 5. RhoA expression is associated with invasion and clinical stages of colorectal cancer. One hundred and fifty cases of colorectal cancer samples were stained with RhoA antibody and graded based on the staining intensity. A representative image of tissue microarray (A) and immunohistochemistry (B). RhoA expression was subdivided depending on invasion and clinical stages (C-E). 
Table II. RhoA expression in colorectal carcinoma tissue.

\begin{tabular}{lccccc}
\hline & \multicolumn{4}{c}{ RhoA expression } & \\
\cline { 2 - 5 } & Negative & + & ++ & +++ & $\begin{array}{l}\text { Positive } \\
\text { rate (\%) }\end{array}$ \\
\hline $\begin{array}{l}\text { No. of } \\
\text { cases }\end{array}$ & 64 & 45 & 34 & 7 & 57.3 \\
\hline
\end{tabular}

Patient samples were stained with RhoA antibody and graded as either negative, low (+), medium (++), or high (+++) expression depending on staining intensity.

(Table III). However, RhoA expression was significantly associated with pT stage, vascular invasion, lymphatic invasion, and clinical stage (Fig. 5C-E and Table III), which is consistent with in vitro data demonstrating defective migration and invasion following RhoA knockdown (Figs. 3 and 4). Specifically, RhoA(+) samples exhibited an approximately 3- to 4-fold higher invasion rate of vascular invasion and lymphatic invasion compared to RhoA(-) samples (vascular invasion, 25.6\% of RhoA(+) versus $7.8 \%$ of RhoA(-); lymphatic invasion, $29.1 \%$ of RhoA(+) versus $10.9 \%$ of RhoA(-); Fig. 5C and D and Table III). In addition, RhoA expression was associated with clinical stages of colorectal cancer; RhoA(-) specimens associated with lower clinical stages, while RhoA(+) specimens had higher clinical stages [ clinical stage I, 20.3\% RhoA(-) versus $5.8 \%$ RhoA(+); clinical stage III, $39.1 \%$ of RhoA(-) versus $52.3 \%$ of RhoA(+); Fig. 5E and Table III]. These results indicate that, as tumors express more RhoA, the disease becomes more invasive, progressing to a higher clinical stage. Finally, we correlated RhoA expression with 5-year survival rate. Using univariate analysis, we found that RhoA expression was significantly associated with patient survival rate. As shown

Table III. The association of clinicopathological features and RhoA expression in colorectal carcinoma samples.

\begin{tabular}{|c|c|c|c|c|}
\hline \multirow[b]{2}{*}{ Clinicopathological factors } & \multicolumn{2}{|c|}{ RhoA } & \multirow[b]{2}{*}{ Total } & \multirow[b]{2}{*}{ P-value } \\
\hline & Positive $(\mathrm{N}=86)$ & Negative (N=64) & & \\
\hline Age, years, mean (SD) & $86(62.01)$ & $64(63.42)$ & & 0.479 \\
\hline Sex, N (\%) & & & & 0.259 \\
\hline M & $50(58.1)$ & $43(67.2)$ & $93(62.0)$ & \\
\hline $\mathrm{F}$ & $36(41.9)$ & $21(32.8)$ & $57(38.0)$ & \\
\hline pT stage, $\mathrm{N}(\%)$ & & & & 0.027 \\
\hline 1 & $0 \quad(0)$ & $4 \quad(6.3)$ & $4 \quad(2.7)$ & \\
\hline 2 & $12(14.0)$ & $10(15.6)$ & $22(14.7)$ & \\
\hline 3 & $61(70.9)$ & $47(73.4)$ & $108(72.0)$ & \\
\hline 4 & $13(15.1)$ & $3(4.7)$ & $16(10.6)$ & \\
\hline pN stage, $\mathrm{N}(\%)$ & & & & 0.245 \\
\hline 0 & $39(45.3)$ & $37(57.8)$ & $76(50.7)$ & \\
\hline 1 & $29(33.7)$ & $19(29.7)$ & $48(32.0)$ & \\
\hline 2 & $18(21.0)$ & $8(12.5)$ & $26(17.3)$ & \\
\hline Distal metastasis, N (\%) & & & & 0.637 \\
\hline$(-)$ & $82(95.3)$ & $62(96.9)$ & $144(96.0)$ & \\
\hline$(+)$ & $4(4.7)$ & $2(3.1)$ & $6(4.0)$ & \\
\hline Vascular invasion, $\mathrm{N}(\%)$ & & & & 0.005 \\
\hline$(-)$ & $64(74.4)$ & $59(92.2)$ & $123(82.0)$ & \\
\hline$(+)$ & $22(25.6)$ & $5 \quad(7.8)$ & $27(18.0)$ & \\
\hline Lymphatic invasion, $\mathrm{N}(\%)$ & & & & 0.007 \\
\hline$(-)$ & $61(70.9)$ & $57(89.1)$ & $118(78.7)$ & \\
\hline$(+)$ & $25(29.1)$ & $7(10.9)$ & $32(21.3)$ & \\
\hline Clinical stage, $\mathrm{N}(\%)$ & & & & 0.045 \\
\hline I & $5(5.8)$ & $13(20.3)$ & $18(12.0)$ & \\
\hline II & $32(37.2)$ & $24(37.5)$ & $56(37.3)$ & \\
\hline III & $45(52.3)$ & $25(39.1)$ & $70(46.7)$ & \\
\hline IV & $4 \quad(4.7)$ & 2 & $6(4.0)$ & \\
\hline
\end{tabular}

Patient samples stained with RhoA antibody were divided into either RhoA-positive or -negative groups. Correlation of clinicopathological factors and RhoA expression was analyzed using a Chi-square test. 


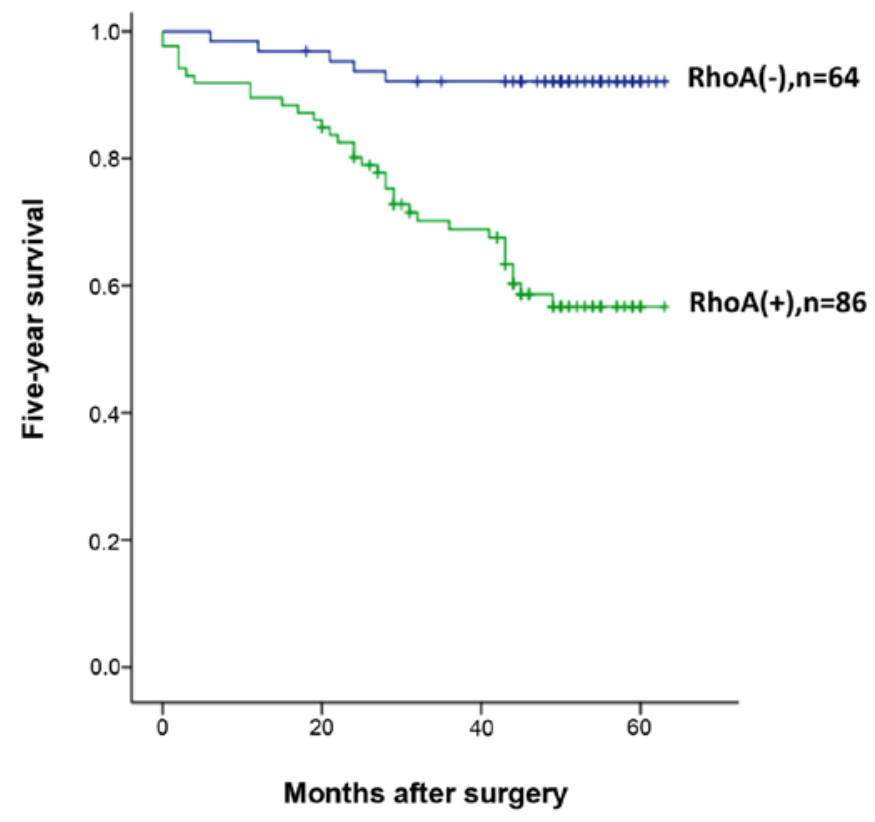

Figure 6. Kaplan-Meier analysis of 5-year survival rate in 150 cases of colorectal cancer. Five-year survival rate of 150 colorectal cancer patients was determined five years post-surgery. RhoA-positive patients had a lower 5-year survival rate than RhoA-negative patients $(\mathrm{p}<0.0001)$, as determined using the Kaplan-Meier method.

in Fig. 6, 92\% of RhoA(-) patients survived whereas only $56 \%$ of $\mathrm{RhoA}(+)$ patients survived five years after surgery. These results indicate that RhoA is associated with invasion and a poor prognosis in colorectal cancer, and could serves as a promising therapeutic target for cancer therapy.

\section{Discussion}

Rho GTPases are small proteins that function as molecular switches in a wide range of systems to transduce signals upon stimulation of cell surface receptors. Signaling through these proteins leads to activation of many relevant pathways in cancer, including cytoskeleton reorganization, proliferation, differentiation, migration, and invasion.

In this study, we investigated the role of RhoA in colorectal cancer using human cell lines as well as 150 patient-derived colorectal cancer samples. First, we found that RhoA is highly expressed in colon cancer cell lines, especially in a metastatic cell line SW620 compared to a non-metastatic cell line SW480. To define the functional importance of RhoA, we generated an shRNA construct against RhoA. We found that, although proliferation of HCT116 cancer cells is only moderately impaired after RhoA knockdown, migration and invasion were significantly reduced in RhoA-depleted HCT116 cells compared to control cells. Furthermore, RhoA(+) cells from colorectal patient samples were more enriched in invasion of lymph node and blood vessels. Moreover, patients with higher expression of RhoA had a significantly poorer 5-year survival rate when followed up for five years after surgery. These results demonstrate that RhoA is important in colorectal cancer, and could be an interesting target for cancer therapeutics.

One of key factors contributing to the mortality in colorectal cancer is metastatic spread of the disease, which is a complex, multistage process. The signal transduction pathway underlying metastasis is not fully known and requires extensive study $(16,17)$. Thus, it is crucial to study signaling pathways contributing to invasion and metastasis in colorectal cancer. In this regard, our results demonstrate that RhoA is strongly associated with invasion and metastasis in colorectal cancer. However, the data we show here is contradictory to recent publications; these publications have shown that lower RhoA expression is correlated with lymph node metastasis in colorectal cancer patients, and that RhoA functions as a tumor suppressor $(14,18)$. Thus, it is important to examine and understand these apparently discrepant results. One of main differences is that we examined colorectal patient samples of different clinical stages whereas Rodrigues et al (14) and Arango et al (18) analyzed only Dukes' stage C. Despite these contradictory findings, many publications indicate that RhoA does play a role in tumor growth and invasion of many types of cancers $(10,13,19,20)$. In addition, RhoA-depleted cancer cells exhibit less proliferation and smaller tumor sizes in vitro and in vivo (21). Furthermore, RhoA is more expressed in tumor samples compared to normal tissues $(10,12,13)$. Overall, it seems that RhoA functions either as a tumor suppressor or activator in a context-dependent manner. As such, it is essential that cancer therapies targeting RhoA should be approached carefully.

In conclusion, our data provide clear evidence implicating RhoA in migration, invasion, and poor prognosis in colorectal cancer. Therefore, this signaling pathway could ultimately serve as a promising target for the treatment and prevention of colorectal cancer metastasis.

\section{Acknowledgements}

This study was supported by the Soonchunhyang University Research Fund and a grant of the Korea Health Technology R\&D Project through the Korea Health Industry Development Institute (KHID), funded by the Ministry of Health \& Welfare, Republic of Korea (grant no. HI15C1647).

\section{References}

1. Jung KW, Won YJ, Kong HJ, Oh CM, Seo HG and Lee JS: Cancer statistics in Korea: Incidence, mortality, survival and prevalence in 2010. Cancer Res Treat 45: 1-14, 2013.

2. Wilke HJ and Van Cutsem E: Current treatments and future perspectives in colorectal and gastric cancer. Ann Oncol 14 (Suppl 2): ii49-ii55, 2003.

3. Chan KM, Wu TH, Cheng CH, Lee WC, Chiang JM, Chen JS and Wang JY: Prognostic significance of the number of tumors and aggressive surgical approach in colorectal cancer hepatic metastasis. World J Surg Oncol 12: 155, 2014.

4. Swiderska M, Choromańska B, Dąbrowska E, KonarzewskaDuchnowska E, Choromańska K, Szczurko G, Myśliwiec P, Dadan J, Ladny JR and Zwierz K: The diagnostics of colorectal cancer. Contemp Oncol (Pozn) 18: 1-6, 2014.

5. Bai Y, Xiang X, Liang C and Shi L: Regulating Rac in the nervous system: Molecular function and disease implication of Rac GEFs and GAPs. BioMed Res Int 2015: 632450, 2015.

6. Stankiewicz TR and Linseman DA: Rho family GTPases: Key players in neuronal development, neuronal survival, and neurodegeneration. Front Cell Neurosci 8: 314, 2014.

7. Karlsson R, Pedersen ED, Wang Z and Brakebusch C: Rho GTPase function in tumorigenesis. Biochim Biophys Acta 1796: 91-98, 2009.

8. Pai SY, Kim C and Williams DA: Rac GTPases in human diseases. Dis Markers 29: 177-187, 2010. 
9. Jaffe AB and Hall A: Rho GTPases: Biochemistry and biology. Annu Rev Cell Dev Biol 21: 247-269, 2005.

10. Wang H, Zhao G, Liu X, Sui A, Yang K, Yao R, Wang Z and Shi Q: Silencing of RhoA and RhoC expression by RNA interference suppresses human colorectal carcinoma growth in vivo. J Exp Clin Cancer Res 29: 123, 2010.

11. Fan YM, Pang CP, Harvey AR and Cui Q: Marked effect of RhoA-specific shRNA-producing plasmids on neurite growth in PC12 cells. Neurosci Lett 440: 170-175, 2008.

12. Liu N, Bi F, Pan YL, Xue Y, Zhang X, Shi YQ, Zhang YM, Du JP and Fan DM: The expression and possible function of RhoA in human gastric cancer cell lines. Zhonghua Zhong Liu Za Zhi 26: 26-29, 2004 (In Chinese).

13. Takami Y, Higashi M, Kumagai S, Kuo PC, Kawana H, Koda K, Miyazaki M and Harigaya K: The activity of RhoA is correlated with lymph node metastasis in human colorectal cancer. Dig Dis Sci 53: 467-473, 2008.

14. Rodrigues P, Macaya I, Bazzocco S, Mazzolini R, Andretta E, Dopeso H, Mateo-Lozano S, Bilić J, Cartón-García F, Nieto R, et al: RHOA inactivation enhances Wnt signalling and promotes colorectal cancer. Nat Commun 5: 5458, 2014.

15. Kodiha M, Umar R and Stochaj U: Optimized immunofluorescence staining protocol to detect the nucleoporin Nup98 in different subcellular compartments protocol exchage, Protocol Exchange: 22 Jan, 2009 (Epub ahead of print). doi:10.1038/ nprot.2009.
16. Chambers AF, Groom AC and MacDonald IC: Dissemination and growth of cancer cells in metastatic sites. Nat Rev Cancer 2: 563-572, 2002.

17. Lee JJ and Lotze MT: Molecular basis of metastasis. N Engl J Med 360: 1679; author reply 1679-1680, 2009.

18. Arango D, Laiho P, Kokko A, Alhopuro P, Sammalkorpi H, Salovaara R, Nicorici D, Hautaniemi S, Alazzouzi H, Mecklin JP, et al: Gene-expression profiling predicts recurrence in Dukes' C colorectal cancer. Gastroenterology 129: 874-884, 2005.

19. Sun K, Duan X, Cai H, Liu X, Yang Y, Li M, Zhang X and Wang J: Curcumin inhibits LPA-induced invasion by attenuating RhoA/ROCK/MMPs pathway in MCF7 breast cancer cells. Clin Exp Med: Jan 18, 2015 (Epub ahead of print).

20. Menhofer MH, Kubisch R, Schreiner L, Zorn M, Foerster F, Mueller R, Raedler JO, Wagner E, Vollmar AM and Zahler S: The actin targeting compound Chondramide inhibits breast cancer metastasis via reduction of cellular contractility. PLoS One 9: e112542, 2014

21. Yang X, Zheng F, Zhang S and Lu J: Loss of RhoA expression prevents proliferation and metastasis of SPCA1 lung cancer cells in vitro. Biomed Pharmacother 69: 361-366, 2015. 